\title{
The aconitate hydratase family from Citrus
}

\author{
Javier Terol, Guillermo Soler, Manuel Talon, Manuel Cercos*
}

\begin{abstract}
Background: Research on citrus fruit ripening has received considerable attention because of the importance of citrus fruits for the human diet. Organic acids are among the main determinants of taste and organoleptic quality of fruits and hence the control of fruit acidity loss has a strong economical relevance. In citrus, organic acids accumulate in the juice sac cells of developing fruits and are catabolized thereafter during ripening. Aconitase, that transforms citrate to isocitrate, is the first step of citric acid catabolism and a major component of the citrate utilization machinery. In this work, the citrus aconitase gene family was first characterized and a phylogenetic analysis was then carried out in order to understand the evolutionary history of this family in plants. Gene expression analyses of the citrus aconitase family were subsequently performed in several acidic and acidless genotypes to elucidate their involvement in acid homeostasis.

Results: Analysis of 460,000 citrus ESTs, followed by sequencing of complete cDNA clones, identified in citrus 3 transcription units coding for putatively active aconitate hydratase proteins, named as CCACO1, CCAcO2 and CCAco3. A phylogenetic study carried on the Aco family in 14 plant species, shows the presence of 5 Aco subfamilies, and that the ancestor of monocot and dicot species shared at least one Aco gene. Real-time RT-PCR expression analyses of the three aconitase citrus genes were performed in pulp tissues along fruit development in acidic and acidless citrus varieties such as mandarins, oranges and lemons. While CCACO3 expression was always low, CCAco 1 and $\mathrm{CCACO} 2$ genes were generally induced during the rapid phase of fruit growth along with the maximum in acidity and the beginning of the acid reduction. Two exceptions to this general pattern were found: 1) Clemenules mandarin failed inducing CCAco2 although acid levels were rapidly reduced; and 2) the acidless "Sucreña" orange showed unusually high levels of expression of both aconitases, an observation correlating with the acidless phenotype. However, in the acidless "Dulce" lemon aconitase expression was normal suggesting that the acidless trait in this variety is not dependent upon aconitases.

Conclusions: Phylogenetic studies showed the occurrence of five different subfamilies of aconitate hydratase in plants and sequence analyses indentified three active genes in citrus. The pattern of expression of two of these genes, CCACO1 and CCACO2, was normally associated with the timing of acid content reduction in most genotypes. Two exceptions to this general observation suggest the occurrence of additional regulatory steps of citrate homeostasis in citrus.
\end{abstract}

\section{Background}

Citrus is one of the most important fruit crops in the world with a total production of 122 million tons in 2008 [1]. Citrus fruits are hesperidium berries with a very special organization composed of two morphologically distinct regions: the pericarp (peel) and the endocarp (pulp), which is the edible portion of the fruit and consists of segments, the ovarian locules, containing the juice vesicles. Growth and development of citrus fruit

\footnotetext{
* Correspondence: cercos_man@gva.es

Centro de Genómica, Instituto Valenciano de Investigaciones Agrarias,

Carretera Moncada - Náquera, Km. 4.5 Moncada (Valencia) E46113, Spain
}

follows a sigmoid curve divided into three stages [2]. After an initial two months interval of slow growth mostly due to cell division (phase I), most of the fruit growth results from cell enlargement and water accumulation during the next 4 to 6 months (phase II). Finally, during phase III, growth is mostly arrested and fruits undergo a non-climacteric ripening process. Research on citrus fruit ripening has received considerable attention because of both the uniqueness of this physiological process and the importance of citrus fruits for the human diet as a supply of ascorbic acid, fibre
() Biomed Central

C 2010 Terol et al; licensee BioMed Central Ltd. This is an Open Access article distributed under the terms of the Creative Commons Attribution License (http://creativecommons.org/licenses/by/2.0), which permits unrestricted use, distribution, and reproduction in any medium, provided the original work is properly cited. 
and several phytochemicals with contrasted benefits for health [3].

Although color break, the pivotal metabolic event characterizing external ripening, takes place during phase III [4,5]; internal quality traits are acquired along both phases II and III [6]. During the first half of phase II, citrus fruits accumulate a considerable amount of organic acids in the vacuoles of the juice sac cells and these acids are progressively catabolized during the second half of phases II and III [7]. The physiological roles of organic acids in fruit cells are not fully understood, although it has been suggested that low $\mathrm{pH}$ could result in an enhanced sink strength, increasing carbohydrate uptake [8]. The characteristic decline in titrable acidity shown by many citrus fruits is due to the utilization of citric acid, the most abundant organic acid in citrus juice [9].

Mature citrus pulp contains a high percentage of water and many other different constituents, including acids and sugars among other determinants of taste and organoleptic quality [10]. Thus, the control of fruit acidity loss has a strong economical relevance since it is related to the consumer perception and hence constitutes a main constraint for the citrus industry. Recent advances in this field resulted in the identification of the main metabolic processes involved in citrate utilization $[7,11,12]$ including a tonoplast citrate $/ \mathrm{H}^{+}$symporter potentially involved in citrate efflux from the vacuole [13]. According to the current hypothesis, indirectly supported by proteomic data [14], citrate is released from the vacuole into the cytosol and then sequentially isomerized into isocitrate by cytosolic aconitase and then metabolized into 2-oxoglutarate by $\mathrm{NADP}^{+}$-isocitrate dehydrogenase. The utilization of 2-oxoglutarate involves transamination into glutamate and then either conversion into glutamine and further utilization for thiamine biosynthesis, or conversion into succinate through the gamma-aminobutirate shunt, eventually leading to carbohydrate synthesis. Alternatively, citrate may be converted to oxaloacetate and acetyl-CoA in a reaction catalyzed by ATP citrate lyase. However, since a decrease in ATP citrate lyase mRNA level during citrus fruit ripening has been reported [7] all steps involved in citrate utilization are initially dependent on its isomerization into isocitrate, the step controlled by aconitase.

Aconitase catalyzes the reversible isomerization of citrate to isocitrate via the intermediate product cisaconitate. Two isoforms of aconitase have been detected in all eukaryotic cells: mitochondrial aconitase that is involved in the tricarboxylic acid cycle, and cytosolic aconitase that participates in several processes, such as cytosolic citrate metabolism $[7,11,12]$ and the glyoxylate cycle $[15,16]$. It is also worth mentioning that aconitases are multifunctional proteins. In addition to the enzymatic activity, it has been shown, for instance, that the yeast mitochondrial aconitase is a component of the mitochondrial nucleoid and interacts with mitochondrial DNA [17], while the cytosolic aconitase has RNA binding activity related to iron homeostasis in animals and to resistance to oxidative stress in both animals and plants $[18,19]$.

The aconitate hydratase family has been only described in detail in Arabidopsis [19,20]. However, the large number of vegetal genomes sequenced to completion in the last years allows performing extensive phylogenetic analysis in these species. Besides the ones from Arabidopsis, rice [21], poplar [22] and vitis [23], released several years ago, the genome sequences from many other plants are now available, and only during 2009 and the beginning of 2010 the genomes of corn [24], sorghum [25], soybean [26] and false purplebrome [27] have been completely sequenced. In this work, we have first characterized the aconitase gene family of citrus and performed, taking advantage of the availability of complete genome sequences, a phylogenetic analysis of the aconitate hydratase family in 14 species, which allowed an unprecedented view of the evolutionary history of this family in plants. In addition, the role of the citrus aconitase genes in the acid homeostasis has been investigated studying their expression in several acidic and acidless citrus genotypes.

\section{Results and discussion}

\section{Molecular characterization of the Citrus aconitate} hydratase genes

In order to identify cDNAs coding for ACO proteins, a BLASTX search was performed against 465,094 citrus ESTs available at the GenBank, using the Arabidopsis thaliana ACO proteins as queries. 179 ESTs produced significant similarity with the Arabidopsis ACO proteins, using an e value cut off of 1e-15. Assembly of the reads with GAP4 resulted in all the ESTs but one clustering into 3 contigs. The only singleton corresponded to a putative immature mRNA with an intron that prevented its assembly. The fact that all the ESTs clustered into 3 unigenes strongly supports that the aconitate hydratase family from citrus is composed, at least, of three transcription units. Three genes have been also reported in A. thaliana, the only plant species in which this family has been described in detail $[19,20]$.

One cDNA clone representing each transcription unit was selected from a normalized full length library constructed from a variety of tissues and organs at different developmental stages and subjected to different abiotic stresses [28]. Clones IC0AAA40BB02RM1, IC0AAA5BE08RM1, and IC0AAA7DC07RM1 were sequenced to completion with a primer walk strategy, resulting in 3 
sequences of 3218, 3331, and 3433 bp respectively, that were submitted to GenBank (Acc\# FN552254, FN552255, and FN552256). Conceptual translation of the cDNA sequences showed that clones ICOAAA 40BB02RM1, IC0AAA7DC07RM1, and IC0AAA5 BE08RM1 contained ORFs coding for proteins of 900, 898, and 898 aa, respectively, named Aco1, Aco 2 and Aco3 genes, based on their similarity with the Arabidopsis ones (see Table 1 for details). An aconitate hydratase gene from Citrus limon that was previously reported [GenBank:AF073507] was 99\% identical to Aco3 from C. clementina.

A multiple alignment of the Arabidopsis and Clementine proteins with ClustalX exhibited a high degree of conservation between the six proteins, with 627 identical residues, and 142 conservative changes, representing an overall similarity higher than $85 \%$. Analysis of the citrus predicted proteins with PFAM [29] showed conservation of the catalytic and the swivel domains [20], suggesting that the citrus proteins are able to catalyze the interconversion of isocitrate and citrate via a cis-aconitate intermediate (Figure 1).

\section{Phylogenetic analysis of the aconitate hydratase family in plants}

In order to study the evolutionary history of the aconitate hydratase family in plants, the complete genome sequences of the dicot species Populus trichocarpa [22], Vitis vinifera [23], Carica papaya [30], Ricinus communis [31], Medicago truncatula [32], and Glycine max [26]; the monocot species Oryza sativa [21], Zea mays [24], Sorghum bicolor [25], and in Brachypodium distachyon [27], and the moss Physcomitrella patens [33] were searched for Aco genes. The BLASTX search identified 3 Aco genes in all but 3 species, which is in agreement with the results from Arabidopsis and citrus. Soybean and poplar displayed 6 and 4 Aco genes, respectively, while only 2 transcription units were discovered in purple false brome. Table 2 shows the Aco ORFs obtained, indicating the species and their accession numbers.

A multiple alignment with 48 Aco sequences was carried out, and a phylogenetic analysis was performed as

Table 1 The Aconitate hydratase genes in Citrus

\begin{tabular}{lccccccc}
\hline & cDNA clone & Acc N $^{\mathbf{0}}$ & bp $^{\mathbf{a}}$ & st $^{\mathbf{b}}$ & end $^{\mathbf{b}}$ & ORF $^{\mathbf{d}}$ & aa $^{\mathbf{e}}$ \\
\hline CCAco1 & ICOAAA40BB02RM1 & FN552254 & 3218 & 103 & 2805 & 2700 & 900 \\
CCAco2 & ICOAAA7DC07RM1 & FN552256 & 3433 & 520 & 3216 & 2694 & 898 \\
CcAco3 & ICOAAA5BE08RM1 & FN552255 & 3331 & 443 & 3139 & 2694 & 898
\end{tabular}

\footnotetext{
${ }^{a} \mathrm{mRNa}$ length in bp

${ }^{\mathrm{b}}$ Start codon position.

'Stop codon position.

dORF length in bp.

e Protein length in aa.
}

described in Methods. The tree obtained (Figure 2) shows six main clusters that include all but one Aco ORFs. As expected, sequences from $P$. patens group in a single cluster with the largest genetic distances respect to the other sequences, which reflects the evolutionary distance between Bryophyta and Angiosperm taxa. All the remaining sequences but Sbi_02g034590, group into 5 clusters, that have been named Aco1, Aco2, Aco3, Aco4, and Aco5, based on the Arabidopsis naming system.

Sequence Sbi_02g034590 from sorghum, annotated as a member of the aconitate hydratase family in the BLAST searches, is not included in the previous clusters. Genetic distances with respect to the other sequences are also in the same range than the ones obtained for $P$. patens suggesting that it should not be considered as part of this family.

Aco1 cluster is composed of sequences from both mono and dicot species, indicating that their ancestor was present in plants previously to the split of mono and dicotyledonous groups. Subclusters Aco2 and Aco3 only gather genes from dicot species, while Aco4-Aco5 clades are formed only by monocot ones. This fact suggests that these clusters appeared after the monocot and dicot split, and have diverged to give rise to new subfamilies. It is noteworthy that all the dicot species, except poplar and soybean, display 3 Aco genes, that are distributed into the Aco1, Aco2 and Aco3 clusters. The organization of the monocot subclusters was found to be similar. Analogous evolutionary patterns with sequences from mono and dicot species distributed among mono and polyphyletic clades have been also found for the polygalacturonase [34], lignin biosynthesis [35], or DOF [36] gene families.

The Aco family in poplar and soybean displays a different organization since both genomes lack Aco3 homologs, and their genes group in Aco1 and Aco2 clusters with very small genetic distances between them, suggesting the occurrence of recent duplication events. In fact, XM_002301587 and XM_002321090 are located on homologous genome blocks from chromosomes II and XIV, produced in a salicoid-specific genome-wide duplication event described in poplar that is still detectable over approximately $92 \%$ of the poplar genome [22]. The salicoid duplication has been also related to the fact that Populus has the largest number of major intrinsic proteins (MIPs) identified in any single plant species [37], with the duplication of several cinnamyl alcohol dehydrogenase (CAD) genes [38], or with the fact that the neutral invertases (NIs) gene family has 9 members in the Arabidopsis genome, 8 in rice, 9 in vitis, while 16 in poplar [39]. Similarly, the 6 Aco genes found in soybean might be originated by succeeding whole-genome duplications, one of them legume specific 13 MYA ago, 


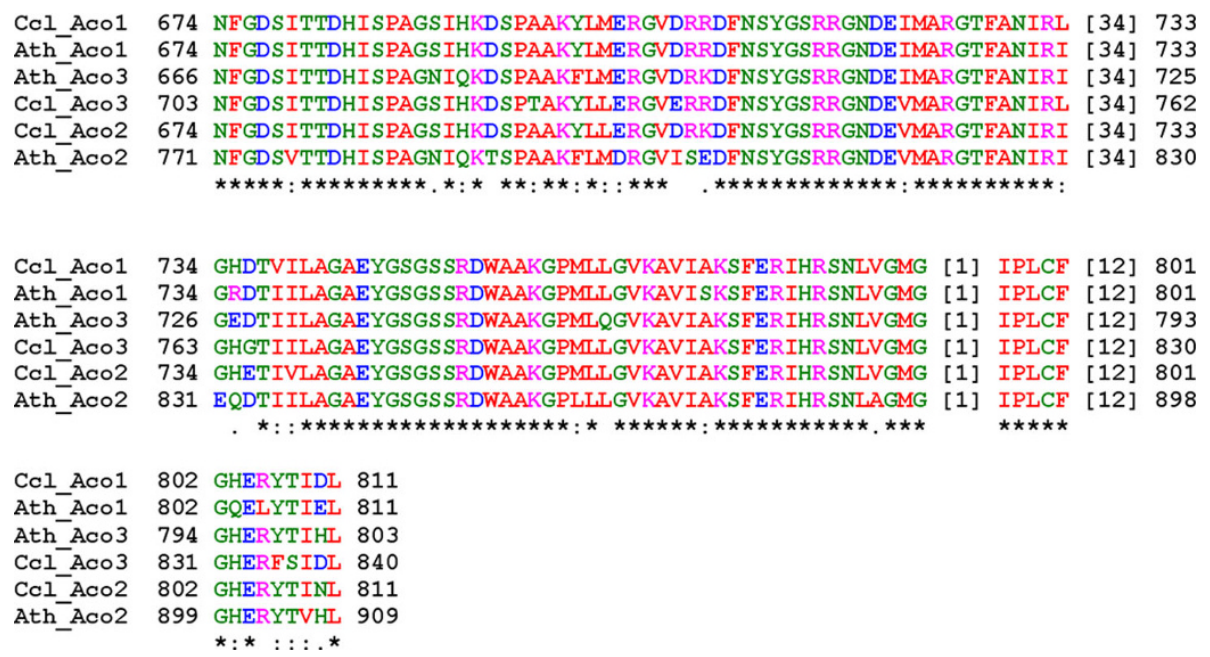

Figure 1 Conservation of the swivel domain in Arabidopsis and Clementine ACO proteins. A multiple alignment of the swivel domain of the aconitate hydratase proteins from Arabidopsis and C. clementina is shown. Asterisks show identical residues in all six sequences, colons indicate conservative changes, and points represent semi-conserved substitutions.

and by an extraordinary level of gene retention occurred in this species, resulting in $75 \%$ of the soybean genes present as multiple copies [26].

In order to find how many of the predicted ORFs were transcriptionally active, a BLAST search against the GenBank EST database was performed with the species analyzed in the phylogenetic study. For each species, the predicted ORFs and the ESTs producing significant scores were assembled with GAP4 (see Methods), which resulted in contigs containing the ORF sequences and all the ESTs associated with each hypothetical gene (Table 2). Considering the large number of analyzed ESTs in several species (1.5 MM in Arabidopsis, 1.2 MM in rice, 260.000 in Medicago, etc), the difference in the number of ESTs found between the Aco genes may reflect the different expression levels of the genes in them.

It is noteworthy that in poplar, with more than 426.000 ESTs analyzed, only one predicted gene per cluster, XM_002327692 (27 ESTs) and XM_002301587 (33 ESTs), show significant levels of expression, while their pairs (XM_002331719, and XM_002321090) only show none and 2 associated ESTs. Chaudhary et al. (2009) described a similar expression asymmetry in the analysis of the carotenoid biosynthesis genes in rice, Arabidopsis and poplar [40]. The analysis of the polygalacturonase gene family in Arabidopsis and rice also showed that tandem duplicated regions had one relatively highly expressed gene while the rest had either low or no expression levels [34], Finally, unequal expression of homologous neutral invertase genes was observed by quantitative RT-PCR in vitis [39]. These changes in the level of expression of duplicated genes have been related with neofunctionalization after gene duplications [41].

\section{Expression of aconitase genes}

To understand the physiological roles of CcAco1, CcAco 2 and $C c A c o 3$ genes and their involvement in the control of fruit acidity levels, their expression in pulp tissues was determined by real-time RT-PCR along fruit development in selected varieties of mandarins, oranges and lemons. Total acidity was also determined in juice extracts to correlate fruit acidity with aconitase gene expression. In mandarins, analyses were performed on two varieties differing in fruit acidity: Clemenules (Citrus clementina Hort. ex Tan. cv. Clemenules) and Fortune (Citrus clementina Hort. ex. Tan. x Citrus reticulata Blanco). Three varieties of sweet orange (Citrus sinensis L. Osb.) were also used: an acidless orange (cv. Sucreña) and two normal acidic ones (cv. Comuna and cv. Valencia Late). The two lemon varieties (Citrus limon L. Burm.) studied were cv. Dulce, that is an acidless lemon and cv. Fino that shows normal acidity levels.

In Clemenules mandarin, total acidity was low during phase I of fruit development (cell division stage), started to increase at the beginning of phase II (cell enlargement stage), reached the maximum levels around 140 days post anthesis and decreased thereafter during the second half of phase II and during phase III (ripening stage). Fortune mandarin acidity showed a similar profile, although acid content was significantly higher at the acidity peak $(69.03 \pm 4.53 \mathrm{mg} / \mathrm{ml}$ in Fortune versus $36.95 \pm 1.40 \mathrm{mg} / \mathrm{ml}$ in Clemenules) and remained higher during the second half of phase II and in mature fruit (Figure 3A), when acid content was $7.74 \pm 0.89 \mathrm{mg} / \mathrm{ml}$ 
Table 2 The Aconitate hydratase genes in plants

\begin{tabular}{|c|c|c|c|c|c|}
\hline Species & ORF Acc. $\mathrm{N}^{\circ}$ & Protein Acc. $\mathbf{N}^{\circ}$ & Locus & Aco class & Associated ESTs \\
\hline \multirow[t]{3}{*}{ A. thaliana } & NM_119749 & NP_195308 & At4g35830 & Acol & 47 \\
\hline & NM_126589 & NP_178634 & At2g05710 & Aco2 & 183 \\
\hline & NM_118831 & NP_567763 & At4g26970 & Aco3 & 131 \\
\hline \multirow[t]{3}{*}{ C. clementina } & FN552254 & CBE71056 & - & Aco1 & 46 \\
\hline & FN552256 & CBE71058 & - & Aco2 & 93 \\
\hline & FN552255 & CBE71057 & - & Aco3 & 35 \\
\hline \multirow[t]{3}{*}{ C. papaya } & DS981532 & - & - & Acol & 7 \\
\hline & DS981607 & - & - & Aco2 & 1 \\
\hline & DS981526 & - & - & Aco3 & 14 \\
\hline \multirow[t]{3}{*}{ M. truncatula } & CR932965_17 & - & МT060719-3729.M00012 & Acol & 61 \\
\hline & AC173287_15 & - & МT060719-3496.M00013 & Aco2 & 40 \\
\hline & AC144481_23 & - & МT060719-2520.M00023 & Aco3 & 2 \\
\hline \multirow[t]{4}{*}{ P. trichocarpa } & XM_002327692 & XP_2327728 & POPTRDRAFT_593790 & Acol & 29 \\
\hline & XM_002331719 & XP_2331755 & POPTRDRAFT_585679 & Acol & 4 \\
\hline & XM_002301587 & XP_2301623 & POPTRDRAFT_816803 & Aco2 & 57 \\
\hline & XM_002321090 & XP_2321126 & POPTRDRAFT_246575 & Aco2 & 5 \\
\hline \multirow[t]{3}{*}{ R. communis } & XM_002530589 & XP_002530635 & RCOM_0782740 & Acol & 2 \\
\hline & XM_002524138 & XP_002524184 & RCOM_0487910 & Aco2 & 12 \\
\hline & XM_002532518 & XP_002532564 & RCOM_0082520 & Aco3 & 0 \\
\hline \multirow[t]{3}{*}{ V. vinifera } & XM_002263301 & XP_2263337 & LOC100242027 & Acol & 29 \\
\hline & XM_002279224 & XP_2279260 & LOC100256776 & Aco2 & 18 \\
\hline & XM_002278102 & XP_2278138 & LOC100253811 & Aco3 & 38 \\
\hline \multirow[t]{6}{*}{ G. $\max$} & BT095399 & & Glyma01g36750 & Acol & 435 \\
\hline & AK286137 & - & Glyma11g08550 & Acol & 61 \\
\hline & - & - & Glyma06g46190 & Aco3 & 38 \\
\hline & AK244974 & - & Glyma12g10580 & Aco3 & 25 \\
\hline & AK286541 & - & Glyma12g32000 & Aco3 & 55 \\
\hline & - & - & Glyma13g38480 & Aco3 & 55 \\
\hline \multirow[t]{3}{*}{ O. sativa } & NM_001055433 & NP_001048898.1 & Loc_Os03g04410 & Acol & 194 \\
\hline & AP005505 & BAD05751 & Loc_Os08g09200 & Aco4 & 216 \\
\hline & NM_001063996 & NP_001057461 & Loc_Os06g19960 & Aco5 & 0 \\
\hline \multirow[t]{4}{*}{ S. bicolor } & XM_002465856 & XP_2465901 & Sb01g047850 & Acol & 17 \\
\hline & XM_002445129 & XP_2445174 & Sb07g005390 & Aco4 & 75 \\
\hline & - & - & Sb06g000210 & Aco5 & 38 \\
\hline & XM_002460720 & XP_2460765 & Sb02g034590 & - & 14 \\
\hline \multirow[t]{2}{*}{ B. distachyon } & - & - & Bradi1g75960 & Acol & 3 \\
\hline & - & - & Bradi3g15050 & Aco4 & 40 \\
\hline \multirow[t]{3}{*}{ Z. mays } & NM_001165757 & NP_001159229 & LOC100304315 & Acol & 188 \\
\hline & NM_001143012 & NP_001136484 & LOC100216599 & Aco4 & 301 \\
\hline & NM_001153959 & NP_001147431 & - & Aco5 & 128 \\
\hline
\end{tabular}

in Clemenules fruits and $27.20 \pm 2.54 \mathrm{mg} / \mathrm{ml}$ in Fortune fruits. Similarly, acidity in Comuna and Valencia Late orange varieties also showed a peak in the middle of phase II at $116 \mathrm{DPA}$, with acidity levels of $49.07 \pm 2.13$ $\mathrm{mg} / \mathrm{ml}$ and $55.47 \pm 2.13 \mathrm{mg} / \mathrm{ml}$ respectively (Figure 3B). Total acidity was extremely low in the acidless Sucreña orange and acidity at 116 DPA was $1.06 \pm 0.05 \mathrm{mg} / \mathrm{ml}$. In cultivar Fino, a normal acidity lemon, acid content also increased during the second half of phase II reaching levels much higher than that of mandarin and orange varieties (for example $85.37 \pm 1.18 \mathrm{mg} / \mathrm{ml}$ at 174 DPA, Figure $3 C$ ). As above, in acidless Dulce lemon, no acidity increase was observed and acid levels remained low and slightly higher than those of Sucreña orange $(4.61 \pm 0.67 \mathrm{mg} / \mathrm{ml}$ at $116 \mathrm{DPA})$.

Quantitative real-time RT-PCR analyses (Figure 4) allowed transcript detection for CcAco1, CcAco 2 and $C c A c o 3$ indicating that these sequences correspond to functional aconitase genes. $C c A c o 3$ was constitutively expressed although at low levels along all phases of fruit 


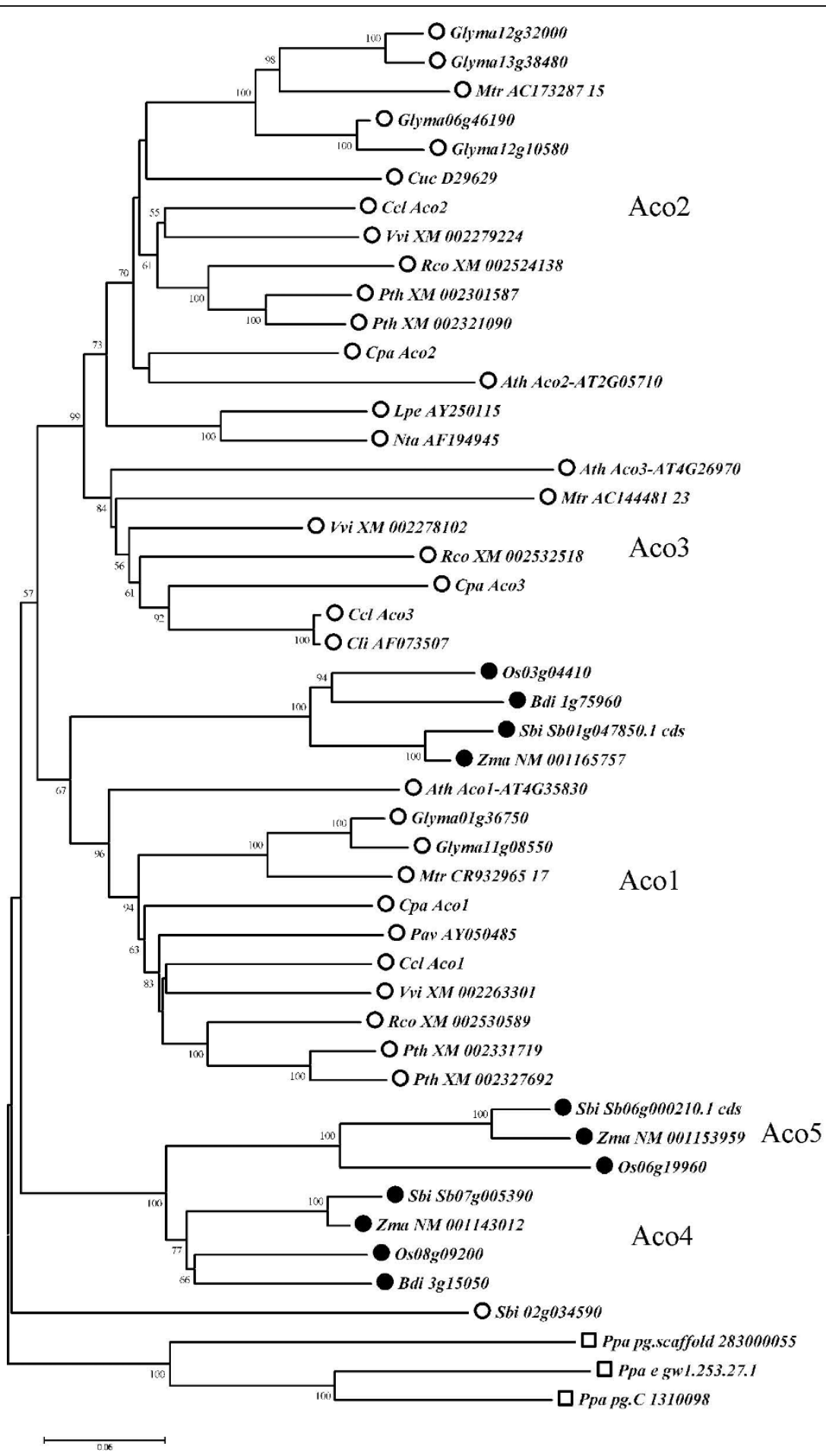

Figure 2 Evolutionary relationships of the aconitate hydratase family in plants. The evolutionary history was inferred using the NeighborJoining method. The optimal tree with the sum of branch length $=4.42716789$ is shown. Only significant bootstrap values are shown next to the branches. The tree is drawn to scale, with branch lengths in the same units as those of the evolutionary distances used to infer the phylogenetic tree. There were a total of 3737 positions in the final dataset. The different Aco subfamilies are indicated with labels close to the corresponding branches. Empty circles indicate dicot species, black circles designate monocot ones, squares mark the bryophyte $P$. patens. Species names are abbreviated as: Ath (Arabidopsis), Bdi (false purplebrome), Ccl (Clementine), Cli (lemon), Cpa (papaya), Cuc (cucumber), Glyma (soybean), Lpe (tomato), Mtr (barrel medic), Nta (tobacco), Osa (rice), Pav (peach), Ppa (P. patens), Pth (poplar), Rco (castor oil), Sbi (sorghum), Vvi (grapevine), and Zma (corn). 


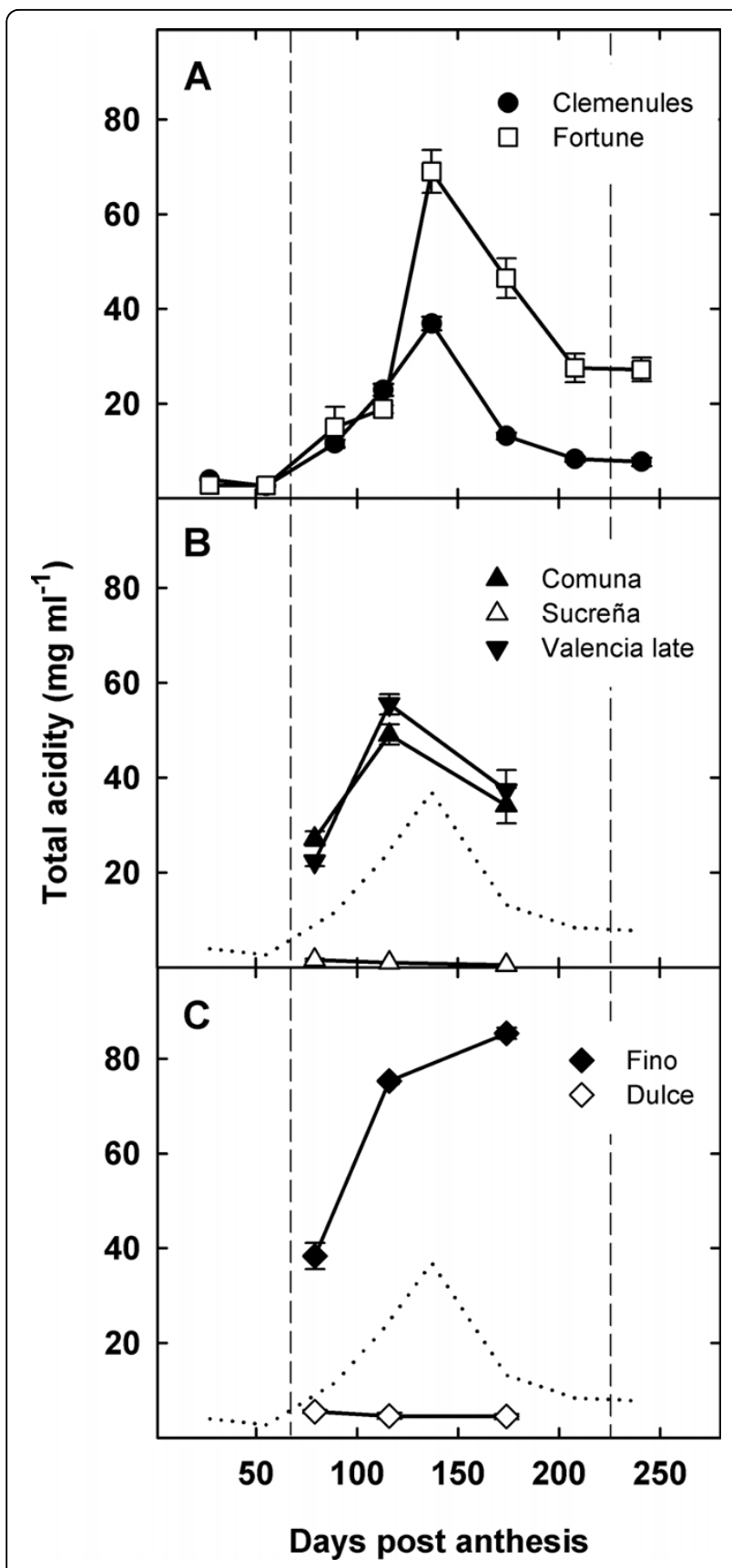

Figure 3 Changes in acidity during fruit development. Total titrable acidity in juice extracts of selected varieties of mandarins $(A)$, oranges (B) and lemons (C) during pulp development.

Clemenules acidity profile (dotted line) is included in panels B and $C$ as a reference. Vertical dashed lines separate the three phases of citrus fruit development: cell division (phase I), cell expansion (phase II) and ripening (phase III).

development in all citrus varieties used for this study (Figure 4C, F, I). Thus, this expression pattern suggests that $C c A c o 3$ would not be deeply involved in the acidity reduction process taking place in the cytosol during pulp ripening and that the CcAco3 protein might have a mitochondrial localization and therefore be involved in the tricarboxylic acid cycle taking place in the mitochondrial matrix. However, cytosolic and mitochondrial compartimentalizations are not mutually exclusive since evidences for the simultaneous localization of plant and yeast aconitase proteins in cytosol and mitochondria have been reported [42].

In general, increase of CcAco 1 and CCAco 2 gene expression started during the first middle part of phase II (Figure 4A, B). Thus, induction of these genes was coincident or slightly preceded the acidity peak while expression was high at the beginning and during the reduction in acid level. This suggests that $C c A c o 1$ and CcAco2 proteins are presumably related to the cytosolic aconitase activity metabolizing the citrate released from the vacuole during ripening $[7,11]$. Interestingly, Clemenules fruits did not show CcAco2 induction, an observation suggesting a possible mutation in the regulatory regions of this gene, since no alteration was detected in the coding sequence (data not shown). In spite of the lack of CcAco 2 induction in Clemenules, acidity and CcAco1 and CcAco 2 mRNA levels in Fortune were still higher than those in Clemenules. Thus, differences in citric acid content between these two varieties may be related to additional regulatory steps such as the activity of the membrane transporters mediating citrate efflux from the vacuole during ripening [13]. Although the mechanisms controlling citrate vacuolar compartmentalization in citrus are not fully understood, several regulatory points have been proposed for the transport of citrate anions across the tonoplast, including a thermodynamically favoured transport through rectifying anion channels [13] and an active ATP-driven transport mechanism [43]. In addition, Shimada et al [13] isolated a novel citrate- $\mathrm{H}^{+}$symporter induced during fruit acidity decrease in Washington Navel. The elucidation of the mechanisms involved in the control of vacuolar citrate compartmentalization may thus help to understand the differences in acidity found between these mandarin varieties.

The highest mRNA levels of CcAco1 and CcAco2 genes were found in the pulp of acidless Sucreña orange and these levels were already high at the onset of phase II, when the expression of these genes was still arrested in normal acidity oranges (Comuna and Valencia Late) mandarins and lemons. The overexpression of these genes when citric acid is being produced in the mitochondrial matrix, released to the cytosol and exported to the vacuole may be responsible for the acidless phenotype of the variety, since a higher aconitase activity would degrade citric acid, avoiding its vacuolar accumulation. 


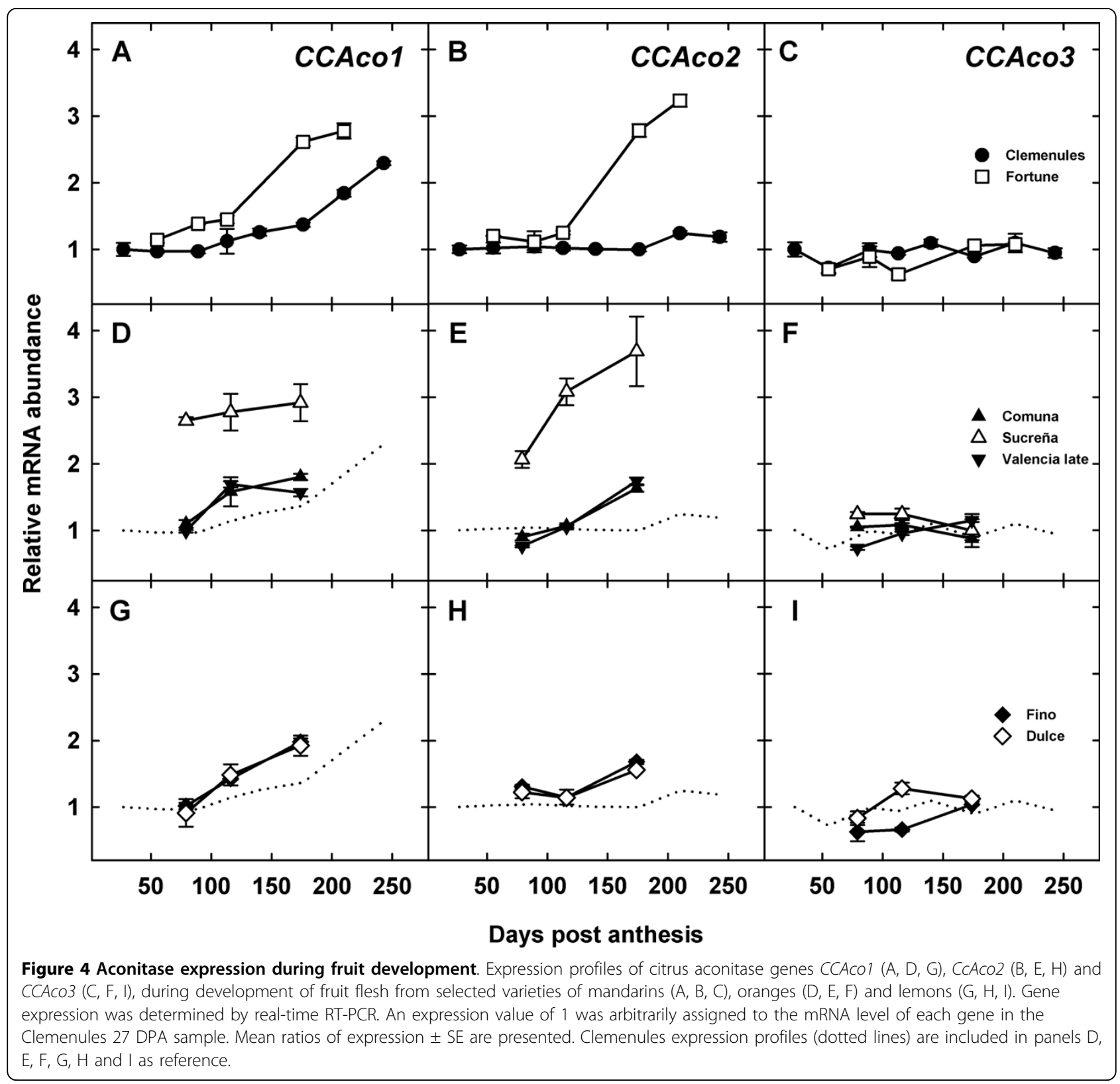

The comparison of Dulce, an acidless lemon variety with Fino, a normal acidity lemon, showed no significant differences in the expression of the putative cytosolic aconitase genes. This suggests that different mechanisms are responsible for the phenotypes of orange (Sucreña) and lemon (Dulce). Furthermore, in limes, a derived species from lemon, an explanation involving additional players has been previously proposed for the acidless trait of sweet lime [44]. Sadka et al. [11] reported that during the initial stages of fruit development a partial inhibition of the enzymatic activity of lemon mitochondrial aconitase allowed the production of high amounts of citric acid in the mitochondria during the initial stages of fruit development. This activity decrease was correlated with a previously reported increase in citramalate, a competitive inhibitor of aconitase activity [44] In acidless sweet lime, citramalate level was lower [44], suggesting that the acidless phenotype of this variety mostly related to citric acid production instead of citric acid removal. This possibility is compatible with the observation that in acidless Dulce lemon, expression of the genes involved in citrate removal was normal (Figure 4 G, H) and expression of one aconitase gene observed at 79 and specially at 116 DPA (Figure 4I) was slightly increased.

An additional finding of this work is that despite the strong accumulation of citric acid in lemons (Figure 
3C), CcAco 2 aconitase mRNA levels were similar to those found in oranges and mandarins (Figure 4G, H). This suggests that citric acid in ripening lemon fruit may not be accessible to the aconitase proteins and reinforces the above idea that regulatory differences in the citrus tonoplast transporters may be relevant to understand the diversity of patterns of citric accumulation and homeostasis in the several citrus fruits.

\section{Conclusions}

Phylogenetic studies showed the occurrence of five different subfamilies of aconitate hydratase genes in plants that derived from one common ancestor of mono and dicots. The analysis of the sequence of the aconitase family in citrus showed that it is composed of three active genes. The pattern of expression of two of these genes, CcAco1 and CcAco2, was normally associated with the timing of citrate reduction in most genotypes including mandarins, oranges and lemons. In addition, two exceptions to this general observation suggest the occurrence of additional regulatory steps of citrate homeostasis in citrus.

\section{Methods}

\section{Plant material}

Parthenocarpic citrus fruits were harvested from adult trees grown in the field under normal cultural practices. The following acidic and acidless varieties and species were used: Clementina mandarin (Citrus clementina Hort. ex Tan.; cv. Clemenules), Fortune mandarin (Citrus clementina Hort. ex. Tan. x Citrus reticulata Blanco), three sweet orange varieties (Citrus sinensis L. Osb.; cv. Comuna, cv. Sucreña and cv. Valencia Late) and two lemon varieties, (Citrus limon L. Burm.; cv. Fino and cv. Dulce). Sucreña orange and Dulce lemon are characterized by a very low level of acid contain and therefore are defined as acidless varieties. Fruits were selected by uniformity of size and appearance, and absence of abiotic and biotic stress symptoms. Homogeneous fruits were peeled and flavedo (exocarp) and albedo (mesocarp) discarded. The remaining tissue, mainly consisting of juice vesicles (endocarp), the segments with their membranes and the vascular bundles, was frozen under liquid nitrogen and stored at $-80^{\circ} \mathrm{C}$ until used.

Two samples of Clemenules were taken during phase I (cell division stage) on May 27, at 27 days post anthesis (DPA), and June 24, at 55 DPA. Five samples were harvested during phase II (cell expansion stage), two before total acidity peak (July 28, 89 DPA, and August 21, 113 DPA), one at maximum acidity (September 17, 140 DPA) and two after the peak of maximum acidity (October 23, 176 DPA and November 26, 210 DPA). The last sample was harvested during phase III (ripening) at the fully ripe stage (December 29, 243 DPA), when acidity content was again low. Samples of Fortune mandarin were harvested at the same times. Fruits of the sweet orange varieties Comuna, Sucreña and Valencia Late, and from the two lemon varieties, Fino and Dulce were harvested during phase II, around the maximum acidity peak of Clemenules, at 79, 116 and 174 DPA. To exclude differences in gene expression due to environmental factors, two sets of samples were taken during the years 2005 and 2008. The data reported here were obtained with the 2005 samples, and similar expression patterns were observed with the 2008 samples (data not shown).

\section{cDNA Sequencing}

Plasmid DNA was extracted from liquid culture (LB plus Amp) with the FastPlasmid Mini Kit (Eppendorf), following manufacturer's instructions. cDNA clones were sequenced to completion by primer walk using the primers shown in additional file 1 Table S1. Sequencing reactions were performed utilizing the ABI Big Dye Terminator Cycle Sequence Ready Reaction as described by manufacturer in an ABI 3730 automatic sequencer. Reads were assembled with the software GAP4 from the Staden package [45], and a consensus sequence was obtained for each transcription unit.

\section{Phylogenetic analysis}

The BLASTX tool was used to discover Aco genes in Populus trichocarpa [22], Vitis vinifera [23], Carica papaya [30], Ricinus communis [31], Medicago truncatula [32], Glycine max [26], Oryza sativa [21], Zea mays [24], Sorghum bicolor [25], and Brachypodium distachyon [27]. The moss Physcomitrella patens [33], was also analyzed as a representative of more primitive taxonomic groups. Additionally, the ORFs of the plant aconitate hydratase proteins from Citrus limon [Genbank: AF073507], Cucurbita sp [GenBank:D29629], Lycopersicon pennelli [GenBank:AY250115], Nicotiana tabacum [GenBank:AF194945], and Prunus avium [Genbank: AY050485], previously described and available at GenBank, were also included in this study.

Although the genome sequence of papaya has been published [30], only DNA sequences produced by the whole genome shotgun (WGS) are accessible at the GenBank. In order to obtain the aconitate hydratase genes from papaya, a BLASTX search was performed against the WGS scaffolds available at the public database. Three scaffolds yielding significant homology were obtained, and ORF prediction was performed with GeneScan [46], followed by manual curation. The 3 ORFs obtained were named Aco1, Aco 2 and Aco 3 based on their similarity with Arabidopsis, and were also included in the phylogenetic analysis. 
Table 3 Oligonucleotides used as primers for real-time RT-PCR

\begin{tabular}{lccc}
\hline Gene & Primer orientation & Primer sequence & Amplicon position \\
\hline CCACO1 & Forward & 5'-GGCAAGTCATTCACATGCGTT-3' & $2692-2852$ \\
& Reverse & 5'-TGAAGAAGTAGACCCCGGTTGA-3' & $2671-2771$ \\
CCACO2 & Forward & 5'-GGCAATGATGAAGTGATGGCT-3' \\
& Reverse & 5'-GTTGGAACATGGACCGTCTIT-3' & $2719-2834$ \\
& Forward & 5'-TGCAGCAATGAGGTACAAGGC-3' \\
\hline
\end{tabular}

A multiple alignment with the 48 predicted Aco ORFs was carried out with Clustal_X [47]. Phylogenetic analyses were conducted in MEGA4 [48]. Genetic distances were calculated with the Maximum Composite Likelihood method [49] and all positions containing alignment gaps and missing data were eliminated only in pairwise sequence comparisons (Pairwise deletion option). There were a total of 3.595 positions in the final dataset. The phylogenetic tree was constructed with the NeighborJoining method [50]. The percentage of replicate trees in which the associated taxa clustered together was calculated with the bootstrap test with 1000 replicates [51]. Similar analyses were performed with the Minimum Evolution [52] and Maximum Likelihood methods [53], and the trees obtained confirmed the clustering obtained with the Neighbor-Joining method (data not shown).

BLASTN [54] search tool was used to identify ESTs associated with the Aco ORFs against the EST section of the GenBank [55]. For each species, the ESTs obtained were assembled with the ORF sequences using GAP4 from the Staden package [45], which allowed counting of the number of identical ESTs associated with an ORF.

\section{Fruit acidity determination}

Fruit acidity was determined by titration of $5 \mathrm{ml}$ of fresh juice extract with $0.1 \mathrm{M} \mathrm{NaOH}$, using phenolphtalein as indicator. Total acidity was used as an indirect measurement of citric acid concentration, the dominant acidic compound in Citrus fruit juice and major responsible of fruit acidity [9].

\section{RNA extraction and real-time RT-PCR}

Total RNA was isolated from frozen tissue using the RNeasy Plant Mini Kit (Qiagen) and treated with RNase-free DNase (Qiagen) according to the manufacturer's instructions. UV light absorption spectrophotometry and agarose gel electrophoresis were performed to test RNA quality as described by Sambrook et al. [56] and RNA concentration was accurately determined by a fluorometric assay with the RiboGreen dye (Molecular Probes) following the manufacturer's instructions. Quantitative real-time RT-PCR was performed with a LightCycler 2.0 Instrument (Roche) equipped with LightCycler Software version 4.0 as described by [7].
One-step RT-PCR was carried out with 25 ng total RNA adding 2.5 units of MultiScribe Reverse Transcriptase (Applied Biosystems), 1 unit RNase inhibitor (Applied Biosystems), $2 \mu \mathrm{l}$ of LC FastStart DNA MasterPLUS SYBR Green I (Roche) and $2.5 \mathrm{pmol}$ of each oligonucleotide in a total volume of $10 \mu \mathrm{l}$. Incubations were carried out at $48^{\circ} \mathrm{C}$ for $30 \mathrm{~min}, 95^{\circ} \mathrm{C}$ for $10 \mathrm{~min}$ followed by 40 cycles at $95^{\circ} \mathrm{C}$ for $20 \mathrm{~s}$, annealing temperature $\left(55^{\circ}\right.$ $\mathrm{C}$ for $C c A c o 1$ and $C c A c o 3$ and $58^{\circ} \mathrm{C}$ for $\left.C c A c o 2\right)$ for $10 \mathrm{~s}$ and $72^{\circ} \mathrm{C}$ for $15 \mathrm{~s}$. Fluorescent intensity data were acquired during the $72^{\circ} \mathrm{C}$ extension step. Gene-specific primers (Table 3) were designed using the Primer Express software (Applied Biosystems). Specificity of the amplification reactions was assessed by postamplification dissociation curves and by sequencing the reaction products. To transform fluorescent intensity measurements into relative mRNA levels, a 10-fold dilution series of a RNA sample was used as standard curve. Reproducible data were obtained after normalization to total RNA amounts [57] since previous work in our lab indicated that other normalization methods rendered irreproducible results [4]. For proper comparison, data were re-scaled so that an induction value of 1 -fold was arbitrarily assigned to the 27 DPA Clemenules sample. Each sample was analyzed in triplicate and mean ratios \pm standard errors were calculated.

\section{Additional material}

Additional file 1: Primers used in cDNA sequencing. This excel file show the oligos used in the primer walk performed to sequence the 3 cDNA clones from C. Clementina.

\section{Acknowledgements \\ Work at the Centro de Genómica (IVIA) was supported by the Spanish Ministerio de Ciencia e Innovación-FEDER grant AGL2007-65437-C04-01/AGR and the INIA grant RTA 2008-00065-00-00.}

\section{Authors' contributions}

$\mathrm{JT}$ carried out characterization of the citrus Aco genes, the phylogenetic analysis in plants and drafted the manuscript. GS performed the RT-PCR analysis. MT and MC coordinated the project and drafted the manuscript. All authors read and approved the final manuscript.

Received: 26 March 2010 Accepted: 19 October 2010 Published: 19 October 2010 
References

1. Food and Agriculture Organization of the United Nations: [http://www.fao. org/corp/topics/en/]

2. Bain JM: Morphological, anatomical, and physiological changes in the developing fruit of the Valencia orange, Citrus sinensis (L) Osbeck. Aust J Bot 1958, 6(1):1-23.

3. Tadeo F, Cercos M, Colmenero-Flores JM, Iglesias DJ, Naranjo MA, Rios G, Carrera E, Ruiz-Rivero O, Lliso I, Morillon R, Talon M: Molecular physiology of development and quality of citrus. Adv Bot Res 2008, 47:147-223.

4. Alos E, Cercos M, Rodrigo MJ, Zacarias L, Talon M: Regulation of color break in citrus fruits. Changes in pigment profiling and gene expression induced by gibberellins and nitrate, two ripening retardants. J Agric Food Chem 2006, 54(13):4888-4895.

5. Alos E, Roca M, Iglesias DJ, Minguez-Mosquera MI, Damasceno CM, Thannhauser TW, Rose JK, Talon M, Cercos M: An evaluation of the basis and consequences of a stay-green mutation in the navel negra citrus mutant using transcriptomic and proteomic profiling and metabolite analysis. Plant Physiol 2008, 147(3):1300-1315.

6. Iglesias DJ, Cercós M, Colmenero-Flores JM, Naranjo MA, Ríos G, Carrera E, Ruiz-Rivero O, Lliso I, Morillon R, Tadeo FR, Talon M: Physiology of citrus fruiting. Braz J Plant Physiol 2007, 19(4):333-362

7. Cercos M, Soler G, Iglesias D, Gadea J, Forment J, Talon M: Global Analysis of Gene Expression During Development and Ripening of Citrus Fruit Flesh. A Proposed Mechanism for Citric Acid Utilization. Plant Mol Biol 2006, 62(4):513-527.

8. Hockema BR, Etxeberria E: Metabolic Contributors to Drought-enhanced Accumulation of Sugars and Acids in Oranges. J Amer Soc Hort Sci 2001 126(5):599-605.

9. Albertini MV, Carcouet E, Pailly O, Gambotti C, Luro F, Berti L: Changes in organic acids and sugars during early stages of development of acidic and acidless citrus fruit. J Agric Food Chem 2006, 54(21):8335-8339.

10. Davies FS, Albrigo LG: Citrus Oxon: CABI Publishing 1994

11. Sadka A, Dahan E, Cohen L, Marsh KB: Aconitase activity and expression during the development of lemon fruit. Physiol Plant 2000, 108:255-262.

12. Sadka A, Dahan E, Or E, Cohen L: NADP(+)-isocitrate dehydrogenase gene expression and isozyme activity during citrus fruit development. Plant Sci 2000, 159(1-2):173-181.

13. Shimada T, Nakano R, Shulaev V, Sadka A, Blumwald E: Vacuolar citrate/H+ symporter of citrus juice cells. Planta 2006, 224(2):472-480.

14. Katz E, Fon M, Lee YJ, Phinney BS, Sadka A, Blumwald E: The citrus fruit proteome: insights into citrus fruit metabolism. Planta 2007, 226(00320935; 4):989-1005

15. Courtois-Verniquet F, Douce R: Lack of aconitase in glyoxysomes and peroxisomes. Biochem J 1993, 294(1):103.

16. Hayashi M, De Bellis L, Alpi A, Nishimura M: Cytosolic aconitase participates in the glyoxylate cycle in etiolated pumpkin cotyledons. Plant Cell Physiol 1995, 36(4):669-680.

17. Chen XJ, Wang $X$, Butow RA: Yeast aconitase binds and provides metabolically coupled protection to mitochondrial DNA. Proc Natl Acad Sci USA 2007, 104(34):13738-13743.

18. Moeder W, Del Pozo O, Navarre DA, Martin GB, Klessig DF: Aconitase plays a role in regulating resistance to oxidative stress and cell death in Arabidopsis and Nicotiana benthamiana. Plant Mol Biol 2007, 63(2):273-287.

19. Arnaud N, Ravet K, Borlotti A, Touraine B, Boucherez J, Fizames C, Briat JF, Cellier F, Gaymard F: The iron-responsive element (IRE)/iron-regulatory protein 1 (IRP1)-cytosolic aconitase iron-regulatory switch does not operate in plants. Biochem J 2007, 405(3):523-531.

20. Peyret $P$, Perez $P$, Alric M: Structure, genomic organization, and expression of the Arabidopsis thaliana aconitase gene. Plant aconitase show significant homology with mammalian iron-responsive elementbinding protein. J Biol Chem 1995, 270(14):8131-8137.

21. International Rice Genome Sequencing Project: The map-based sequence of the rice genome. Nature 2005, 436(7052):793-800

22. Tuskan GA, DiFazio S, Jansson S, Bohlmann J, Grigoriev I, Hellsten U, et al: The Genome of Black Cottonwood, Populus trichocarpa (Torr. \& Gray). Science 2006, 313(5793):1596-1604.

23. Jaillon O, Aury JM, Noel B, Policriti A, Clepet C, Casagrande A, et al: The grapevine genome sequence suggests ancestral hexaploidization in major angiosperm phyla. Nature 2007, 449(7161):463-467.
24. Schnable PS, Ware D, Fulton RS, Stein JC, Wei F, Pasternak S, et al: The B73 Maize Genome: Complexity, Diversity, and Dynamics. Science 2009, 326(5956):1112-1115.

25. Paterson AH, Bowers JE, Bruggmann R, Dubchak I, Grimwood J, Gundlach $\mathrm{H}$, et al: The Sorghum bicolor genome and the diversification of grasses. Nature 2009, 457(7229):551-556

26. Schmutz J, Cannon SB, Schlueter J, Ma J, Mitros T, Nelson W, et al: Genome sequence of the palaeopolyploid soybean. Nature 2010 463(7278):178-183.

27. The International Brachypodium Initiative: Genome sequencing and analysis of the model grass Brachypodium distachyon. Nature 2010, 463(7282):763-768

28. Terol J, Conesa A, Colmenero JM, Cercos M, Tadeo FR, Agusti J, Alos E, Andres F, Soler G, Brumos J, Iglesias DJ, Gotz S, Legaz F, Argout X, Courtois B, Ollitrault P, Dossat C, Wincker P, Morillon R, Talon M: Analysis of 13000 unique Citrus clusters associated with fruit quality, production and salinity tolerance. BMC Genomics 2007, 8:31.

29. Finn RD, Tate J, Mistry J, Coggill PC, Sammut SJ, Hotz H, Ceric G, Forslund K, Eddy SR, Sonnhammer ELL, Bateman A: The Pfam protein families database. Nucl Acids Res 2008, 36(suppl_1):D281-288.

30. Ming R, Hou S, Feng Y, Yu Q, onne-Laporte A, Saw JH, et al: The draft genome of the transgenic tropical fruit tree papaya (Carica papaya Linnaeus). Nature 2008, 452(7190):991-996.

31. Castor Bean Genome Database. [http://castorbean.jcvi.org]

32. Cannon SB, Sterck L, Rombauts S, Sato S, Cheung F, Gouzy J, et al: Legume genome evolution viewed through the Medicago truncatula and Lotus japonicus genomes. Proc Natl Acad Sci USA 2006, 103(40):14959-14964.

33. Rensing SA, Lang D, Zimmer AD, Terry A, Salamov A, Shapiro $H$, et al: The Physcomitrella genome reveals evolutionary insights into the conquest of land by plants. Science 2008, 319(5859):64-69

34. Kim J, Shiu S, Thoma S, Li W, Patterson S: Patterns of expansion and expression divergence in the plant polygalacturonase gene family. Genome Biol 2006, 7(9):R87

35. Xu Z, Zhang D, Hu J, Zhou X, Ye X, Reichel $K$, et al: Comparative genome analysis of lignin biosynthesis gene families across the plant kingdom. BMC Bioinformatics 2009, 10:S3.

36. Yang X, Tuskan GA, Cheng ZM: Divergence of the Dof Gene Families in Poplar, Arabidopsis, and Rice Suggests Multiple Modes of Gene Evolution after Duplication. Plant Physiol 2006, 142(3):820-830.

37. Gupta A, Sankararamakrishnan R: Genome-wide analysis of major intrinsic proteins in the tree plant Populus trichocarpa: Characterization of XIP subfamily of aquaporins from evolutionary perspective. BMC Plant Biology 2009, 9(1):134

38. Barakat A, Bagniewska-Zadworna A, Choi A, Plakkat U, DiLoreto DS, Yellanki $P$, Carlson JE: The cinnamyl alcohol dehydrogenase gene family in Populus: phylogeny, organization, and expression. BMC Plant Biology 2009, 9:26.

39. Nonis A, Ruperti B, Pierasco A, Canaguier A, Adam-Blondon AF, Di Gaspero G, Vizzotto G: Neutral invertases in grapevine and comparative analysis with Arabidopsis, poplar and rice. Planta 2008, 229(1):129-142.

40. Chaudhary N, Nijhawan A, Khurana JP, Khurana P: Carotenoid biosynthesis genes in rice: structural analysis, genome-wide expression profiling and phylogenetic analysis. Mol Genet Genomics 2010, 283:13-33.

41. Innan $\mathrm{H}$, Kondrashov $\mathrm{F}$ : The evolution of gene duplications: classifying and distinguishing between models. Nat Rev Genet 2010, 11(2):97-108.

42. Carrari F, Nunes-Nesi A, Gibon Y, Lytovchenko A, Loureiro ME, Fernie AR: Reduced expression of aconitase results in an enhanced rate of photosynthesis and marked shifts in carbon partitioning in illuminated leaves of wild species tomato. Plant Physiol 2003, 133(3):1322-1335.

43. Canel C, Bailey-Serres JN, Roose ML: In Vitro [14C]Citrate Uptake by Tonoplast Vesicles of Acidless Citrus Juice Cells. J Amer Soc Hort Sci 1995, 120(3):510-514.

44. Bogin E, Wallace A: Organic acid synthesis and accumulation in sweet and sour lemon fruit. J Am Soc Hort Sci 1966, 89:182-194.

45. Staden R: The Staden sequence analysis package. Mol Biotechnol 1996 5(3):233-241

46. Burge $C B$, Karlin S: Finding the genes in genomic DNA. Curr Opin Struct Biol 1998 , 9(3):346-354.

47. Larkin MA, Blackshields G, Brown NP, Chenna R, McGettigan PA McWilliam $H$, Valentin F, Wallace IM, Wilm A, Lopez R, Thompson JD, 
Gibson TJ, Higgins DG: Clustal W and Clustal X version 2.0. Bioinformatics 2007, 23(21):2947-2948.

48. Tamura K, Dudley J, Nei M, Kumar S: MEGA4: Molecular Evolutionary Genetics Analysis (MEGA) software version 4.0. Mol Biol Evol 2007, 24(0737-4038; 8):1596-1599.

49. Tamura $\mathrm{K}, \mathrm{Nei} \mathrm{M}$, Kumar $\mathrm{S}$ : Prospects for inferring very large phylogenies by using the neighbor-joining method. Proc Natl Acad Sci USA 2004, 101(30):11030-11035

50. Saitou N, Nei M: The neighbor-joining method: A new method for reconstructing phylogenetic trees. Mol Biol Evol 1987, 4:406-425.

51. Felsenstein J: Confidence limits on phylogenies: An approach using the bootstrap. Evolution 1985, 39:783-791.

52. Rzhetsky A, Nei M: Theoretical foundation of the minimum-evolution method of phylogenetic inference. Mol Biol Evol 1993, 10(5):1073-1095.

53. Felsenstein J: Evolutionary trees from DNA sequences: a maximum likelihood approach. J Mol Evol 1981, 17(6):368-376.

54. Altschul SF, Gish W, Miller W, Myers EW, Lipman DJ: Basic local alignment search tool. J Mol Biol 1990, 215(3):403-410

55. Benson DA, Karsch-Mizrachi I, Lipman DJ, Ostell J, Wheeler DL: GenBank: update. Nucl Acids Res 2004, 32:D23-D26.

56. Sambrook J, Fritsch E, Maniatis T: Molecular Cloning Cold Spring Harbor Laboratory Press, Cold Spring Harbor, N.Y 1989.

57. Hashimoto JG, Beadles-Bohling AS, Wiren KM: Comparison of RiboGreen and $18 \mathrm{~S}$ rRNA quantitation for normalizing real-time RT-PCR expression analysis. BioTechniques 2004, 36(1):58-60, 54-6.

doi:10.1186/1471-2229-10-222

Cite this article as: Terol et al.: The aconitate hydratase family from

Citrus. BMC Plant Biology 2010 10:222.

\section{Submit your next manuscript to BioMed Central and take full advantage of:}

- Convenient online submission

- Thorough peer review

- No space constraints or color figure charges

- Immediate publication on acceptance

- Inclusion in PubMed, CAS, Scopus and Google Scholar

- Research which is freely available for redistribution

Submit your manuscript at www.biomedcentral.com/submit 